\title{
Clinical Utility of Bladder Cancer Biomarkers
}

\author{
Laura-Maria Krabbe, ${ }^{\boxplus 1}$ Georgios Gakis, ${ }^{2}$ Yair Lotan ${ }^{3}$ \\ ${ }^{1}$ The University of Muenster Medical Center, Germany, ${ }^{2}$ University Hospital of Würzburg, Germany, \\ ${ }^{3}$ The University of Texas Southwestern Medical Center, United States
}

\begin{abstract}
Each year, there are an estimated 550000 diagnoses of bladder cancer worldwide, and almost 200000 deaths from bladder cancer. The need for frequent follow-up, including invasive procedures like cystoscopy, repetitive procedures like transurethral resection of bladder tumors and intravesical instillation therapy in non-muscle invasive stages, as well as systemic treatment with or without radical local treatment in advanced stages, makes bladder cancer one of the most expensive cancers to treat. Prognostic and predictive biomarkers have the potential to fundamentally change bladder cancer treatment algorithms, which may result in improved patient comfort and oncological outcomes and may also decrease the socioeconomic burden of the disease. Intense research has resulted in the recent approval by the U. S. Food and Drug Administration of the first agent for this disease that targets a specific mutation (fibroblastgrowth factor receptor). Yet, many areas of bladder cancer diagnosis and treatment have remained unchanged for decades, and this is only in part due to their therapeutic success. In order to integrate biomarkers into clinical practice patterns, specific considerations for the different disease stages and settings should be kept in mind. Especially in the setting of screening, work-up of hematuria, as well as surveillance of patients with non-muscle invasive bladder cancer, (urine-)biomarkers may prove useful. They must, however, demonstrate a high enough sensitivity to pick up a cancer diagnosis or recurrence, allow easy handling (preferably a point-of-care setting) and adequate cost-benefit relationships, while also providing additional information to a full work-up. A biomarker to identify patients with muscle invasive bladder cancer who are in need of - and likely to respond to-neoadjuvant therapy would be very useful. In later disease, early detection of recurrence or progression, as well as biomarkers guiding treatment decisions between the available systemic agents, will be paramount for improved patient care.
\end{abstract}

\section{Introduction}

There are about 550000 new diagnoses of bladder cancer each year, and it was responsible for almost 200000 deaths worldwide in 2018 [1]. The need for frequent follow-up, including invasive procedures like cystoscopy, repetitive procedures like transurethral resection of bladder tumors and intravesical instillation therapy in non-muscle invasive stages, as well as systemic treatment with or without radical local treatment in advanced stages, makes bladder cancer one of the most expensive cancers to treat [2]. Prognostic and predictive biomarkers have the potential to fundamentally change bladder cancer treatment algorithms, which may improve patient comfort and oncological outcomes, as well as decreasing the socioeconomic burden of the disease. The U. S. Food and Drug Administration has recently approved the first agent for this disease that targets a specific mutation [3]. Yet, many aspects of bladder cancer diagnosis and treatment have remained unchanged for decades, and this is due only in part to their therapeutic success. In order to integrate biomarkers into clinical practice patterns, specific considerations for the different disease stages and settings should be kept in mind.

\section{Key Words}

Biomarkers, bladder cancer, muscle invasive bladder cancer, non-muscle invasive bladder cancer, neoadjuvant therapy, hematuria, cystoscopy, risk stratification, clinical decision-making

\section{Competing Interests}

None declared.

\section{Article Information}

Received on July 14, 2020

Accepted on August 3, 2020

Soc Int Urol J 2020; 1(1): 62-67 


\begin{tabular}{ll}
\multicolumn{2}{l}{ Abbreviations } \\
BCG & bacillus Calmette-Guérin \\
MIBC & muscle invasive bladder cancer \\
NMIBC & non-muscle invasive bladder cancer \\
NAC & neoadjuvant chemotherapy \\
NLST & National Lung Screening Trial \\
NPV & negative predictive value \\
PD-L1 & programmed death receptor ligand 1 \\
PLCO & Prostate, Lung, Colorectal and Ovarian Cancer \\
& Screening Trial \\
PPV & positive predictive value \\
RC & radical cystectomy
\end{tabular}

\section{Considerations for Biomarkers for Bladder Cancer Screening}

Because of the low incidence of bladder cancer in the general population (and even in high-risk populations), there is currently no recommendation for bladder cancer screening [4]. In general, for screening purposes in daily routine, a point-of-care test on a dichotomous basis could be performed easily in the ambulatory setting and should provide an initial risk assessment which could be used to tailor the need for further clinical investigation. Urine is obviously the most promising resource for such a test. On the one hand, a urine-based biomarker test requires a high sensitivity and negative predictive value (NPV) to ensure people at risk are not missed. On the other hand, given the low incidence, the test also needs a high specificity and positive predictive value (PPV) to prevent unnecessary (invasive) evaluation that would occur because of false positive results. In addition, given its application of a urine marker as a screening tool, the costs for test analysis should be low and not greatly exceed the usual costs for standard diagnostic work-up of bladder cancer. Finally, an earlier detection after test analysis should ideally demonstrate a significant improvement in oncological outcomes. As it seems to be difficult to demonstrate an overall survival benefit in the general-or even high-risk-population, a reduction of rates of invasive disease may serve as a surrogate parameter since there are known significant differences in survival between non-muscle invasive bladder cancer (NMIBC) and muscle invasive bladder cancer (MIBC). Most current urine markers have a PPV that is too low to justify their use in screening since the number of unnecessary evaluations will far exceed the finding of cancer. Targeted screening of very high-risk populations may result in a high enough cancer incidence, but prospective studies will be necessary to demonstrate a survival benefit or at least a reduction of muscle invasive disease. It is known that male sex, older age, and duration and intensity of smoking are associated with higher risk of bladder cancer. A study evaluating these factors and incidence of bladder cancer in the Prostate, Lung, Colorectal and Ovarian (PLCO) Cancer Screening Trial and the National Lung Screening Trial (NLST) found that in men older than 70 years with smoking exposure of $\geq 30$ pack-years, incidence rates were as high as 11.92 (PLCO) and 5.23 (NLST) per 1000 person-years [5]. There is not a strong association between family history and bladder cancer. However, in the presence of distinct single-nucleotide polymorphisms that are associated with a significant risk for bladder cancer, screening for bladder cancer may be justified. Upper tract urothelial carcinoma develops in up to $28 \%$ of patients with known Lynch syndrome. These patients may be candidates for screening and routine urine analysis, as well as possible further evaluation using the American Urological Association guideline of $\geq 3$ red blood cells per high power field [6]. Patients with Lynch syndrome who develop upper tract disease usually present at a younger age, with a higher female preponderance and a predisposition to bilaterality.

\section{Diagnosis in Patients With Hematuria}

Patients with hematuria have a markedly increased risk of having bladder cancer (gross $10 \%$ to $40 \%$, depending on other risk factors, or microscopic $2 \%$ to $5 \%$ ) and therefore need further clinical work-up $[7,8]$. This work-up consists of cystoscopy with cytology, as well as contrast-enhanced imaging of the upper urinary tract [4]. These procedures are costly, invasive, or uncomfortable. Therefore it would be desirable to replace them with a biomarker test to spare patients who do not need a full evaluation. Again, urine seems to be the most promising medium for a biomarker test in such a scenario. Large efforts have been undertaken to stratify risk in patients with hematuria based on demographic and clinical factors as well as various genetic and protein markers [4]. Dichotomous or semiquantitative tests indicating the individual risk for bladder cancer seem suitable to guide clinicians towards or against further testing. As with screening, a high sensitivity and NPV is needed to ensure a tumor is not missed. A high specificity and PPV are also desirable to prevent unnecessary evaluations for false positive test results, but are less important than in screening, given the lower number of these "quasi"pre-screened patients and related costs.

Since the rate of cancer in patients with hematuria greatly exceeds the rate in screening cohorts, identifying high-risk patients who always need evaluation is important. Several studies have found that the cancer yield increases with gross hematuria, male sex, and increasing age $[9,10]$. Unfortunately, many patients with high-risk disease are not adequately evaluated [11-13]. While the incidence of urothelial carinoma in patients 
with gross hematuria already justifies evaluation, risk stratification to select patients for work-up might benefit patients with microscopic hematuria. The goal for markers could be to improve risk stratification of patients so that higher risk patients get evaluation, but there is also identification of which lower risk patients need to be evaluated and which patients may be safely monitored without invasive testing $[14,15]$. A prospective randomized trial is currently open that randomizes patients based on clinical risk and marker status (NCT03988309). Patients in the control arm will have standard evaluation while those in the marker arm will have a clinical risk stratification, so patients with low clinical risk and a negative marker will not have cystoscopy but follow-up only, but those with a positive marker or higher risk based on clinical factors will undergo a standard evaluation with cystoscopy. Further studies of this type will be necessary to change guideline recommendations.

\section{Surveillance of Patients With Non-Muscle Invasive Bladder Cancer}

When patients are diagnosed with low- to intermediaterisk NMIBC, the follow-up course is usually associated with frequent cystoscopies to rule out recurrent or progressive disease, especially in those undergoing intravesical instillation therapy. These examinations are cumbersome to many patients and are associated with significant costs. Therefore, investigators have focused on biomarkers to accurately detect the presence or absence of a recurrence as an alterative to invasive diagnostic procedures. Given the low risk of progression in low- to intermediate-risk NMIBC, $(<15 \%$ at 5 years), but high risk of recurrence, the goals for a biomarker need to be considered. A biomarker with a high specificity will reduce the number of cystoscopies performed because of false positive findings, but if sensitivity is not high, it may miss recurrences [16]. This may be acceptable in order to reduce the number of cystoscopies since low-grade recurrences are unlikely to progress. As an alternative, some investigators have proposed alternating cystoscopy with a marker. This is more practical, as most markers have a better sensitivity for high-grade disease and would therefore be more likely to catch the rare case of a low-grade tumor that progresses. Furthermore, periodic cystoscopy would assure patients and urologists that even if a marker missed a low-grade recurrence then the cancer would be detected at subsequent evaluation. The UroFollow study, for example, was developed as a prospective randomized trial to compare routine cystoscopy with a urine markerguided, noninvasive follow-up of patients with $\mathrm{pTa}$ G1-2/low-grade NMIBC [17].

On the other hand, patients with a history of highrisk bladder cancer have the highest risk of recurrence
(50\% at 5 years) [16]. Therefore, a useful biomarker test must have a high sensitivity and NPV in order not to miss any high-grade recurrence which may result in progression to muscle-invasive stages. This could even come at the cost of lower test specificity. Ideally a marker would be able to detect cancers missed by white light cystoscopy, which is known to miss some high-grade cancers, especially carcinoma in situ [18]. Unfortunately, urine marker studies have not been designed to assess whether a patient with a normal cystoscopy and positive marker have a true positive or false positive finding. It is therefore not clear what steps should be taken if a marker assay is positive in this scenario.

Molecular markers could also aid in clinical decisionmaking for patients with NMIBC in predicting response to intravesical therapy. Adding a biomarker test to a standard work-up may identify patients who are unlikely to benefit from BCG bacillus Calmette-Guérin and require early radical treatment. Nonetheless, this issue is difficult to address in clinical trials, as bladder cancer is a heterogeneous disease, and the intravesical therapies used are non-specific to a distinct molecular target for which testing would be possible. Therefore, predicting response to intravesical therapy will remain challenging in the future. This is also complicated by the fact that immunotherapy with BCG and also chemoinstillation induce inflammatory changes, which can sometimes impair the diagnosis of a tumor recurrence or influence test results. However, adding a biomarker test to a standard work-up may identify patients unlikely to benefit from BCG, who might require more radical treatment, early [19]. Furthermore, markers may be able to select populations at higher risk for enrollment in clinical trials [20].

\section{Biomarkers With Muscle Invasive Bladder Cancer}

In MIBC, there are several distinct needs that could be addressed by biomarkers. Patients with AJCC stages 2 and 3 disease have variable rates of recurrence and progression. Furthermore, approximately $40 \%$ of patients are understaged [21]. There is level 1 evidence for use of neoadjuvant chemotherapy (NAC), but it is underused because of concerns about toxicity and a relatively small survival benefit [22]. There is a greater benefit to NAC in patients who have non-organ confined disease, so identifying patients likely to have micrometastatic disease would be valuable. Since there is also variable response to NAC, a marker to identify likely responders would be important to prevent toxic treatments being given to patients unlikely to benefit from them.

Several possible biomarkers have been described, including mutations in DNA repair genes $A T M, R B 1$, 
and FANCC, [23] mutations in excision repair crosscomplementation group 2 (ERCC2) gene, [24,25] protein biomarkers, [26] and RNA subtyping of bladder cancer $[27,28]$.

Furthermore, while cystectomy is the main treatment for MIBC, trimodality therapy is a reasonable alternative in some patients, and predicting responsiveness to this type of treatment would be valuable.

In patients who do not get NAC, there is a role for adjuvant therapy in patients with non-organ confined disease [29].

In addition, proponents of an adjuvant approach argue that there is a considerable risk of overtreatment with NAC, since histopathological risk factors determined in radical cystectomy (RC) specimens have a much stronger correlation to survival than histological parameters obtained by transurethral resection of bladder tumors $[30,31]$. Pathological complete or partial response (downstaging to non-muscle invasive tumor stages) after NAC is reported in $40 \%$ to $50 \%$ of the patients and associated with excellent survival [32]. On the contrary, the majority of patients will exhibit persistent muscle-invasive disease ( $\geq \mathrm{ypT} 2)$ after RC, which is associated with poor outcomes [32].

A predictive biomarker in this setting should be capable of identifying patients in need of systemic treatment and those whose tumors are sensitive to systemic treatment (maybe in future even indicate which treatment). Given the high degree of intratumoral heterogeneity of MIBC [33], biomarker expression levels should be homogenous within the tumor lesion to reliably predict response. Ideally, a biomarker assessed in the transurethral resection specimen should have a higher sensitivity than specificity to prevent undertreatment of MIBC patients. By contrast, a high specificity but low sensitivity may result in the underuse of neoadjuvant treatment, but this may be alleviated if an effective adjuvant treatment is available that is safe even in patients with comorbidities (eg, impaired renal function). The currently available histopathological parameters (ie, tumor and nodal stage) can be considered accurate enough to determine the necessity of adjuvant treatment in case of lack of a downstaging effect after neoadjuvant therapy; however, a biomarker might help in determining the most appropriate treatments in the future [34].

Prediction of response in the adjuvant setting after failure of a neoadjuvant approach ideally requires a biomarker with an even higher specificity than the (primary) neoadjuvant setting, because the group of $\geq$ ypT2 patients after RC exhibit a very dismal prognosis [32].
For patients with a locally advanced tumor after RC who have not received previous neoadjuvant therapy, there might be benefit in a prognostic biomarker indicating which patients will experience recurrence and which will not, as well as a predictive biomarker of which treatment might be associated with the best response.

\section{Metastatic Bladder Cancer}

In the metastatic cancer/disease, predictive biomarkers are urgently needed to determine which tumor will likely respond to which treatment, as this will help ensure patients in this very aggressive disease state are not receiving ineffective therapies. These biomarkers will likely be blood- or tissue-based. Ideally, biomarkers should be assessable sequentially via blood draw so the patient does not have to undergo multiple biopsies and the biomarker provides an accurate representation of tumor characteristics, given that tumors, but especially metastases, evolve over time. Groundwork on these fronts has been done by genomic characterization of bladder tumors; there are, however, very few validated biomarkers.

Biomarkers to predict response to therapy are critical. There is already an approved targeted therapy for metastatic bladder cancer targeting fibroblastgrowth factor receptor mutations [35]. The role for biomarkers such as programmed death receptor ligand 1 (PD-L1) to predict response to checkpoint inhibitors is controversial but necessary in certain cases. In firstline cisplatin-ineligible patients who are eligible for carboplatin, the use of PD-L1 inhibitors is currently approved only after PD-L1 testing, whereas in the second-line, data from a randomized trial support the use of for example pembrolizumab in an unscreened population of platinum-pretreated patients $[36,37]$. Given the low response rates in both settings $(25 \%$ complete and partial remissions), it will be important to combine PD-L1 inhibitors with other targeting agents to improve response. Nonetheless, in the future, it can be expected that the decision to use single- or multiagent targeted therapy in any line of systemic therapy will be based on marker expression. Given the high mutational burden and heterogeneity of response to treatment in metastasized tumors, the critical question is whether the tissue obtained at primary diagnosis can accurately reflect the tumor biology after multiple lines of systemic treatment. Therefore, the implementation of robust biomarkers in the different metastatic settings will require first a better understanding of the biological processes during progression of metastatic disease. This will require well-designed biopsy studies to systematically assess alterations in tumor biology during the process of metastasis formation. 


\section{Conclusion}

There are many areas in bladder cancer where biomarkers can have an important role in improving clinical decision-making. The current information from stage and grade of disease is insufficient to adequately

\section{References}

1. Burger M, Catto JW, Dalbagni G, et al., Epidemiology and risk factors of urothelial bladder cancer. Eur Urol 2013; 63: 234-241.

2. Elias K, Svatek RS, Gupta S, et al., High-risk patients with hematuria are not evaluated according to guideline recommendations. Cancer 2010; 116: 2954-2959.

3. Garg T, Pinheiro LC, Atoria CL, et al., Gender disparities in hematuria evaluation and bladder cancer diagnosis: a population based analysis. J Urol 2014; 192: 1072-1077.

4. Ghandour R, Freifeld Y, Singla N, Lotan Y. Evaluation of hematuria in a large public health care system. Bladder Cancer 2019; 5: 119-129.

5. Lotan Y, Svatek RS, Krabbe LM, et al., Prospective external validation of a bladder cancer detection model. J Urol 2014; 192: 1343-1348.

6. Lotan Y, Capitanio U, Shariat SF, et al., Impact of clinical factors, including a point-of-care nuclear matrix protein-22 assay and cytology, on bladder cancer detection. BJU Int 2009; 103: 1368-1374.

7. Sylvester RJ, van der Meijden AP, Oosterlinck W, et al., Predicting recurrence and progression in individual patients with stage Ta T1 bladder cancer using EORTC risk tables: a combined analysis of 2596 patients from seven EORTC trials. Eur Urol 2006; 49: 466-465; discussion 475-467.

8. Benderska-Soder N, Hovanec J, Pesch B, et al., Toward noninvasive follow-up of low-risk bladder cancer-Rationale and concept of the UroFollow trial. Urol Oncol 2020.

9. Daneshmand S, Patel S, Lotan Y, et al., Efficacy and safety of blue light flexible cystoscopy with hexaminolevulinate in the surveillance of bladder cancer: a phase III, comparative, multicenter study. J Urol 2018; 199: 1158-1165.

10. Kamat AM, Willis DL, Dickstein RJ, et al., Novel fluorescence in situ hybridization-based definition of bacille Calmette-Guerin (BCG) failure for use in enhancing recruitment into clinical trials of intravesical therapies. BJU Int 2016; 117: 754-760.

11. Lotan $Y$, Inman BA, Davis LG, et al., Evaluation of the fluorescence in situ hybridization test to predict recurrence and/or progression of disease after bacillus calmette-guerin for primary high grade nonmuscle invasive bladder cancer: results from a prospective multicenter trial. J Urol 2019; 202: 920-926.

12. Shariat SF, Palapattu GS, Karakiewicz PI, et al., Discrepancy between clinical and pathologic stage: impact on prognosis after radical cystectomy. Eur Urol 2007; 51: 137-149; discussion 149-151. stage or predict outcomes for most patients. Biomarkers have the potential to shed light onto clinical behavior of tumors to allow a personalized approach to care. There is also a potential for improved understanding of the biology of the disease to determine which patients need more intensive therapy and which therapies to use.

13. Alfred Witjes J, Lebret T, Comperat EM, et al., Updated 2016 EAU Guidelines on Muscle-invasive and Metastatic Bladder Cancer. Eur Urol 2017; 71: 462-475.

14. Plimack ER, Dunbrack RL, Brennan TA, et al., Defects in DNA repair genes predict response to neoadjuvant cisplatin-based chemotherapy in muscle-invasive bladder cancer. Eur Urol 2015; 68: 959-967.

15. Liu D, Plimack ER, Hoffman-Censits J, et al., Clinical validation of chemotherapy response biomarker ERCC2 in muscle-invasive urothelial bladder carcinoma. JAMA Oncol 2016; 2: 1094-1096.

16. Van Allen EM, Mouw KW, Kim P, et al., Somatic ERCC2 mutations correlate with cisplatin sensitivity in muscle-invasive urothelial carcinoma. Cancer Discov 2014; 4: 1140-1153.

17. Baras AS, Gandhi N, Munari E, et al., Identification and validation of protein biomarkers of response to neoadjuvant platinum chemotherapy in muscle invasive urothelial carcinoma. PLoS One 2015; 10: e0131245.

18. Choi W, Porten S, Kim S, et al., Identification of distinct basal and luminal subtypes of muscle-invasive bladder cancer with different sensitivities to frontline chemotherapy. Cancer Cell 2014; 25: $152-165$.

19. Seiler R, Ashab HA, Erho N, et al., Impact of molecular subtypes in muscle-invasive bladder cancer on predicting response and survival after neoadjuvant chemotherapy. Eur Urol 2017.

20. Advanced Bladder Cancer Meta-analysis C. Adjuvant chemotherapy in invasive bladder cancer: a systematic review and metaanalysis of individual patient data Advanced Bladder Cancer (ABC) Meta-analysis Collaboration. Eur Urol 2005; 48: 189-199; discussion 199-201.

21. Gakis G, Efstathiou J, Lerner SP, et al., ICUD-EAU International Consultation on Bladder Cancer 2012: Radical cystectomy and bladder preservation for muscle-invasive urothelial carcinoma of the bladder. Eur Urol 2013; 63: 45-57.

22. Kukreja JB, Porten S, Golla V, et al., Absence of tumor on repeat transurethral resection of bladder tumor does not predict final pathologic TO stage in bladder cancer treated with radical cystectomy. Eur Urol Focus 2018; 4: 720-724.

23. Zargar H, Zargar-Shoshtari K, Lotan Y, et al., Final pathological stage after neoadjuvant chemotherapy and radical cystectomy for bladder cancer-does pT0 predict better survival than $\mathrm{pTa} / \mathrm{Tis} / \mathrm{T} 1$ ? J Urol 2016; 195: 886-893. 
24. da Costa JB, Gibb EA, Nykopp TK, et al., Molecular tumor heterogeneity in muscle invasive bladder cancer: Biomarkers, subtypes, and implications for therapy. Urol Oncol 2018.

25. Witjes JA, Babjuk M, Bellmunt J, et al., EAU-ESMO Consensus Statements on the Management of Advanced and Variant Bladder Cancer-An International Collaborative Multistakeholder Effort(dagger): Under the Auspices of the EAU-ESMO Guidelines Committees. Eur Urol 2019.

26. Loriot $Y$, Necchi A, Park SH, et al., Erdafitinib in locally advanced or metastatic urothelial carcinoma. N Eng/ J Med 2019; 381: 338-348.
27. Balar AV, Castellano D, O'Donnell PH, et al., First-line pembrolizumab in cisplatin-ineligible patients with locally advanced and unresectable or metastatic urothelial cancer (KEYNOTE-052): a multicentre, single-arm, phase 2 study. Lancet Oncol 2017; 18: 1483-1492.

28. Bellmunt J, de Wit R, Vaughn DJ, et al., Pembrolizumab as second-line therapy for advanced urothelial carcinoma. N Engl J Med 2017; 376: 1015-1026. 